\section{Bre 15}

THE DIAGNOSTIC VALUE OF THE $45^{\circ}$ MEDIOLATERAL PROJECTION IN MAMMOGRAPHY

R. Eulenburg, G. Lauth. D. Drögemeier

In the present work, the $45^{\circ}$ medio-lateral (m-I) projection mammography, is compared with the commonly used cranio-caudal $(\mathrm{c}-\mathrm{c})$ and medio-lateral (m-l) projections. In a retrospective study we compared 500 known cancer with respect to their visibility in the different projections. Visualized in $21 ? 3$ projections 438 $m-1$ and $45^{\circ}$ projections $\mathrm{c}-\mathrm{C}$ and $45^{\circ}$ projections $c-c$ and $m-1$ projections Only in the $45^{\circ}$ projection only in the m-1 projection only in the c-c projection We cound that $95,4 \%$ of all cancers coull seen in the $45^{\circ}$ projection.

In a prospective stualy, we looked it another 500 mammographies. This time only considering the $45^{\circ}$ projection. We then compared these results $\left(45^{\circ}\right)$ with those of the radiologist (all 3 projections).

Insus- Suspicion Suspision ControI picious of benign of maligne

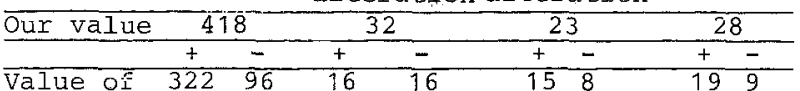

\section{Radiologist}

We diagnosed 15 (75\%) of 20 cancers and evalua-

ted $2(10 \%)$ others as suspicious (together $85 \%$ ).

Three cases (15\%) were described as insuspicious.

Frauenklinik der Philipos-Universität Marburg D-3550 Narburg, Pilgrimstein 3

\section{Bre 16}

RESULTS AND EXPERIENCES WITH STEREOTAXIC FINE NEEDLE BIOPSY (SFNB)

\section{H.J. Gent, E. Sprenger}

In breast cancer the prognosis is influenced decisively by the size of tumor and by whether metastases have spread to the axillary lymph nodes. To diagnose tumors that are less than 1 $\mathrm{cm}$ in size clinical examiniation and palpation are rather crude procedures with limited value. In order to solve those problems with nonpalpable breast lesions the method of SFNB was developed in Sweden in 1977. Our experiences with this Mammotest- system exist since 1983. To date 543 patients have been referred. In 53 cases $(9.8 \%)$ the biopsy could not be performed for methodological reasons. of 490 patients, $38.2 \%$ $(n=187)$ were subjected to SFNB and then operated on after preoperative stereotaxic chromolocalization. Taken together, $78.6 \%(n=147)$ of these cases showed benign histologies. Including one false negative cytological report. malignancy was found in $21.4 \%(n=40)$. Morphometrically, 20 of these tumors were smaller than $5 \mathrm{~mm}$. One control group ( $31.6 \%$ ) was subjected to repeated biopsies and mammography. A second control group $(32.8 \%)$ was followed up only mammographically. In both groups the findings were unchanged after two years. The statistical results (cytology vs. histological examination, Chi-square-test) of 187 patients showed a sensitivity of $97.5 \%$ and a specifity of $95.2 \%$. This is equivalent to the standard achieved in the diagnosis of cervical cancer, which is generally considered optimal. It follows from this that SFNB is a method of high validity, easy to perform, not very timeconsuming, and well tolerated by all patients. It is a valuable addition to mammography.

Department of Gynecology and Obstetrics of the University of Kiel, Hegewischstr. 4, D-2300 Kiel

\section{Bre 17}

CYTOKERATIN ANTIBODIES IN THE EARLY DETECTION OF BONE MARROW INVOLVEMENT IN BREAST CANCER C.Manegold,B.Krempien, K. Schwechheimer, M. Kaufmann

The purpose of our study was to investigate the value of cytokeratin antibodies for early confirmation of bone marrow involvement in breast cancer.Since September 1984 we have obtained bone marrow for cytological and histological evaluation from 50 patients during primary operative treatment bilaterally from the anterior iliac crest. In a11 cases no previous evidence of distant metastases had been found. The histological examination was done on undecalcified bone marrow sections. The immuecytochemical studies were carried out on interphase preparations of bone marrow aspirates (an average of 6 preparations per patient and side). For staining cytokeratin antibodies(PKK 1, Lab System, Helsinki) and the immunealkaline-phosphatase-method were used. Keratin positive cells were found in 4 of 50 cases $(8 \%)$. 2 of the immuecytologically positive cases already showed histological evidence of neoplastic bone marrow infiltration (4\%). The keratin positive cases were characterized as follows:

$\mathrm{T}_{1} \mathrm{~N}_{\mathrm{pos}}(1 / 24) \mathrm{M}_{0}, \mathrm{ER}-, \mathrm{PR}-$, ductal invasive carcinoma

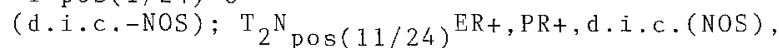

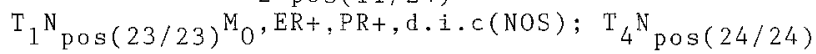
M ,ER+,PR-,d.i.c.( Iobular type). Our study was able to prove that immunecytochemistry is superior to conventional histology in confirming tumor cells in bone marrow. Our results, however, did fall clearly below the $28 \%$ described by Redding et.aI.(1983) with EMA - antibodies.

Medizinische Universitätsklinik, Bergheimer Str.58 D - 6900 Heidelberg.

\section{Bre 18}

ESTROGEN RECEPTORS IN BONE MARROW BIOPSIES OF BREAST CANCER METASTATIC TO THE SKELETION K. Klinga, C. Manegold, P. Krempien and M. Kaufmann The usefulness of receptor determination for the prediction of human breast cancer hormone dependency is well documented. Breast cancer metastases also contain receptors and much data exist concerning their presence in lymph nodes. The purpose of this study was to investigate estrogen receptors of bone marrow metastases in patients with breast cancer. 22 women with skeletal metastases confirmed by bone scan/or $x$-ray were biopsied on the anterior iliac crest using the Jamshidi technique. The tissue obtained sufficed for the determination of estrogen receptors (ER) as well as for histological examination. Cytosolic ER was calculated from a five point Scatchard plot according to the recommendations of the EORTC. The histology of the primary tumor was known in 20 cases (19 ductal invasive carcinoma, 1 adenoid cystoid carcinoma). The steroid receptor status of the primary tumor had been determined in 13 cases. The majority of the women $(n=15)$ had previously received a variety of antihormonal treatment (tamoxifen, medroxyprogesterone acetate, ovarectomy). Results: 10 of the 22 cases showed histological evidence of bone marrow metastases. The concentrations of ER ranged from minor 1 to $20.0 \mathrm{fmol} / \mathrm{mg}$ protein, which can be considered negative or borderline concentrations. There was no significant difference between the patients with bone metastases (median ER 9.5 f mol/mg protein) and those without (median ER $2.9 \mathrm{fmol} / \mathrm{mg}$ protein). Because of the small number of patients, the low ER content and the similar incidence of $\mathrm{ER}$ in marrow with and without metastases, it is difficult to classify groups with hormone sensitive or insensitive tumors. Kan et al.(1984) demonstrated the absence of steroid receptors in the epiphysial cartilage of dogs and rabbits. This finding supports the hypothesis that the physiological effects of estradiol on skeletal growth seem to be a secondary effect without hormone-receptor interaction. On the other hand the low ER may be explained by changes in the receptor status due to the patients' previous hormonal treatment.

Univ.Frauenklinik, Voßstr. 9, D-6900 Heidelberg, West-Germany. 